\title{
Edad de inicio y modelos clínicos en la Esquizofrenia
}

\author{
Zack Z. Cernovsky \\ Johan A. Landmark* \\ Richard L. O'Reilly
}

Departamento de Psiquiatría, Universidad

de Western Ontario, London, Ontario,

CANADA

* Fallecido, el 21 de marzo de 1992

\begin{abstract}
RESUMEN - Se estudio la relación existente entre la edad de inicio (E.I.) y los modelos clínicos en 112 pacientes diagnosticados de esquizofrenia según los criterios DSM III. Empleamos los coeficientes de correlación para controlar la duración de la enfermedad y evitar así el factor de confusión de la cronicidad.

Los pacientes con inicio tardío de la enfermedad presentaron un mayor número de delirios paranoides aunque mostrando problemas relacionados con la volición (pérdida de la iniciativa o energía) en menos ocasiones. Estos pacientes con inicio tardío de la enfermedad tenían una mayor tendencia a presentar síntomas de otro tipo de enfermedad mental distinta a la esquizofrenia. Todas las correlaciones fueron débiles (los valores absolutos de $r$ iban de, 21 a ,24, p < ,05) y no superarían la corrección de Bonfernoni.
\end{abstract}

\section{Introducción}

Según los primeros estudiosos de esta enfermedad, la esquizofrenia solía iniciarse en el curso de la adolescencia o en el inicio de la edad adulta. De acuerdo con los datos de Bleuler, aproximadamente entre el 83$85 \%$ de los pacientes tenían su primer brote de esquizofrenia antes o en torno a los 40 años. En aquellos otros casos en los que el inicio era más tardío podían presentar diferentes cuadros clínicos, especialmente en lo que respecta a la sintomatología paranoide.
Angst Baastrup, Grof, Hippius, Poldinger, Varga, Weis, and Wyss (1973) descubrieron que el $35 \%$ de los casos de esquizofrenia paranoide se iniciaron después de los 40 y un $13 \%$ tras los 50 años.

Con respecto al pronóstico, se ha sugerido que los pacientes situados en los extremos, es decir, los que presentaban mayor limitación o una recuperación más completa, estaban relacionados con el inicio precoz de la enfermedad; por el contrario, los pacientes con inicio tardío solían presentar un pronóstico más homogéneo con recuperaciones 
menos frecuentes y deterioros menos graves (Hamilton 1984).

La duración de la enfermedad podría ser un factor de confusión si comparamos la sintomatología de los pacientes de inicio tardío con los de inicio precoz. Esta fuente de confusión puede ser conceptualizada clínicamente como cronicidad y ser controlada estadísticamente de diferentes formas. Por ejemplo, el procedimiento de comparar los casos de inicio precoz con los tardíos con respecto a la duración de la enfermedad tiene la gran ventaja de que es muy fácil de entender, incluso por aquellos médicos que no tienen grandes conocimientos estadísticos. En cualquier caso, también se puede obtener información estadística similar partiendo de datos que no hayan sido comparados. Todo ello mediante la correlación parcial de coeficientes en los que la relación existente entre la edad y los síntomas se calcule mediante la duración de la enfermedad.

Este procedimiento de correlación parcial se aplicó en el presente estudio con el fin de determinar si existía correlación entre la edad de inicio (E.I.) y determinados cuadros clínicos del espectro de la esquizofrenia. Hemos empleado coeficientes parciales para controlar la duración de la enfermedad. Más concretamente hemos investigado las relaciones entre la edad de inicio (E.I.) y la escala de los 87 síntomas clínicos (Landmark 1982) que son fundamentales para la evaluación de la esquizofrenia. Nuestros coeficientes de correlación en estos análisis fueron controlados estadísticamente con respecto a la duración de la enfermedad.

\section{Método}

Los pacientes que cumplían criterios DSM-III de esquizofrenia fueron 112 (42 hombres y 70 mujeres). Todos ellos habían sido tratados con neurolépticos orales y en el momento en que se puso en marcha este estudio se encontraban estabilizados gracias a tratamientos depot como el decanoato o el enantato de flufenacina. Todos ellos eran pacientes del Hospital Psiquiátrico de Londres, Londres (Ontario, Canadá). Esta muestra está compuesta por pacientes incluidos en un grupo que habían participado en otros estudios sobre la esquizofrenia (Cernovsky, Landmark \& Leslie 1985, Helmes,Landmark \& Kazarian 1983, Landmark 1982; Landmark, Cernosvsky, Merskey \& Leslie 1986, Landmark, Merskey, Cernovsky \& Helmes 1990). Se excluyeron aquellos pacientes que poseían un bajo nivel de inteligencia o padecían síndromes cerebrales orgánicos o déficits sensorio motrices.

Los síntomas de los pacientes fueron tipificados empleando una versión modificada de la lista diagnóstica de Landmark (1982). El listado completo de los 87 síntomas que se utilizaron en este estudio (ver apéndice) ha sido publicado en otros trabajos por Cernovsky y Landmark (1994).

Este listado de síntomas estaba basado en entrevistas estructuradas con pacientes y familiares así como en la información proporcionada por las historias clínicas:los 112 pacientes fueron valorados mediante entrevistas individuales. Los datos sobre "acuerdo interexaminadores"de los síntomas listados para este estudio, así como los datos de frecuencias relativas de los síntomas ya fueron publicados en otros estudios (Helmes et al. 1983; Landmark et al. 1990)

En el análisis de los datos, todos los síntomas de esquizofrenia fueron codificados como presentes (puntuación=1) cuando se manifestaron al menos una vez en el pasado o existían en la actualidad. Cuando el síntoma no aparecía o era dudoso éste se clasificaba como ausente (puntuación=2). 


\section{Resultados}

\section{Edad de inicio (E.I.)}

La edad de los pacientes en esta muestra oscilaba entre los 20 y los 65 años (una media de $38,1, \mathrm{SD}=9,8$ años, con una mediana de 37 años). La edad, en el momento del primer ingreso a causa de la esquizofrenia se situaba entre los 16 y los 59 años de edad (una media de 25,7, SD =7,2, con una mediana de 24 años). En este estudio se ha utilizado la edad en que se produjo el primer ingreso como estimación aproximada del momento de inicio de los síntomas de la esquizofrenia (E.I.). Pues bien, la mayoría de nuestros pacientes (el 95,5\%) de esta muestra fueron hospitalizados por vez primera a causa de la esquizofrenia antes de los 40 años. Es mas, la mayor parte de ellos $(78,6 \%)$ fueron hospitalizados como consecuencia de esta enfermedad antes de los 30 años.

\section{Relación entre la edad de inicio (E.l.) y los síntomas corregidos por la cronicidad}

La relación entre E.I. y los síntomas de la esquizofrenia se calculó mediante una matriz de 87 coeficientes de correlación parcial. En estos coeficientes, las relaciones que se establecen entre la edad de inicio de los pacientes y los síntomas se controlaron por la duración de la enfermedad (teniendo en cuenta que dicha duración se calculaba teniendo en cuenta el número de años desde el que se produjo el primer ingreso hasta el momento de la investigación). Ninguno de los 87 coeficientes fue significativo en $\mathrm{p}=$ ,01 (2-tailed). Con el fin de evitar posibles críticas por haber descartado los aspectos clínicos mas débiles debido a los estrictos criterios de significación empleados, nosotros examinamos también los coeficientes significativos al ,05. Se encontraron así las siguientes correlaciones: $\mathrm{p}<0,05$ o $\mathrm{p}=0,05$ (2-tailed). Aquellos pacientes cuyo primer ingreso tuvo lugar a una edad más avanzada presentaban delirios paranoides $(\mathrm{r}=, 24, \mathrm{p}=$ ,011) aunque ellos mostraron menos frecuentemente problemas relacionados con la afectación volitiva $(\mathrm{r}=, 19, \mathrm{p}=, 025)$. En este estudio, los síntomas volitivos se identificaron a través de manifestaciones tales como pérdida de la iniciativa, de la energía, o simplemente falta de voluntad (véase Landmark 1982). Los pacientes cuyo primer ingreso se produjo a una edad avanzada mostraban con una mayor frecuencia síntomas de otras enfermedades mentales diferentes a los de la esquizofrenia $(r=, 23, p=, 016)$.

\section{Relación entre la edad de inicio (E.I.) y los síntomas no corregidos por la cronicidad}

Puede que los datos respecto a la cronicidad que no han sido corregidos se aproximen más a la realidad clínica. De nuevo calculamos una matriz de 87 coeficientes de correlación parcial para evaluar las relaciones establecidas entre la edad de inicio y los síntomas de la esquizofrenia. Ninguno de $\operatorname{los} 87$ coeficientes fue significativo para una $p=0,01$ (2-tailed). Con el fin de evitar posibles críticas por emplear criterios de significación rigurosos que menospreciaran los aspectos mas débiles, los estudiamos con coeficiente de 0,05 y descubrimos que existían una serie de relaciones con $\mathrm{p}<0,05 \mathrm{o} p$ $=0,05$ (2-tailed). Por un lado los pacientes cuyo primer ingreso se realizó a edad avanzada presentaban con mayor frecuencia delirios paranoides $(\mathrm{r}=, 23, \mathrm{p}=, 015)$ y con menor frecuencia problemas con la actividad volitiva $(\mathrm{r}=, 21, \mathrm{p}=, 031)$ y "bloqueos del pensamiento" $(\mathrm{r}=, 19, \mathrm{p}=, 048)$. En este estudio,se definieron los "bloqueos del pen- 
samiento" como aquellos pensamientos que terminan inesperadamente, de manera tal que la mente se queda en blanco, como si las ideas hubieran sido "robadas" (véase Landmark 1982). También mostraban con más frecuencia síntomas propios de enfermedades psiquiátricas diferentes de la esquizofrenia $(r=, 23, p=, 017)$.

\section{El nivel educativo y el sexo como posibles elementos de confusión}

Los estudios sobre diferencias sexuales generalmente indican que el sexo masculino está relacionado con una aparición más temprana de la esquizofrenia (Torrey 1989). Sin embargo, en nuestra muestra, la edad media de inicio resultó ser muy parecida $(24,9, \mathrm{SD}=7,5$ para los hombres $\mathrm{y}$ $26,1, \mathrm{SD}=7,0$ para las mujeres). La correlación entre sexo y edad de inicio estaban cercanas a cero $(\mathrm{r}=, 08, \mathrm{p}=, 391)$, lo que indicaba que el sexo tenía muy poca influencia. Nuestros hallazgos sobre diferencias entre ambos sexos ya habían sido señalados en otros estudios (Cernovsky, Landmark y O’Reilly 1997).

La correlación entre la edad de inicio y el nivel educativo (basado en las categorías educativas utilizadas por Cernovsky, Landmark y Helmes 1994) era significativa pero débil $(\mathrm{r}=, 21, \mathrm{p}=, 033)$. Nuestros análisis anteriores de esta misma muestra de pacientes, revelaron que el nivel educativo tan solo estaba relacionado de una forma muy superficial con los síntomas de la esquizofrenia, sin encontrar correlación con ninguno de los síntomas que tradicionalmente han sido considerados como marcadores específicos de la esquizofrenia (Cernovsky, Landmark y Helmes 1994). En resúmen, ni el sexo ni el nivel educativo parecían ser decisivos en estos casos.

\section{Discusión}

Solamente 4 (el 3,6\%) del total de nuestros 112 pacientes de esquizofrenia fueron ingresados por primera vez a causa de esta enfermedad después de haber cumplido los 40 años. Es posible que estos 4 pacientes hubieran mostrado algún síntoma antes de los 40, pero no llegaron a recibir atención médica.

Cuando se controlaron nuestros datos estadísticamente a fin de calcular la duración de la enfermedad, los pacientes con comienzos más tempranos de la esquizofrenia, a menudo presentaban síntomas paranoides y dificultades volitivas, sin embargo no eran propensos a presentar síntomas de otros trastornos psiquiátricos diferentes a los de la esquizofrenia. Los hallazgos en torno a los síntomas paranoides coinciden con estudios anteriores (Angst et al. 1973) en el sentido de que, en general los síntomas paranoides se observan con más frecuencia en pacientes con inicio tardío de la enfermedad.

Cuando nuestros datos sobre la edad de inicio se calculan sin ninguna corrección estadística, en lo que respecta a la duración de la enfermedad, se obtiene un modelo de correlación casi idéntico. La única y mínima diferencia detectada fue el descubrimiento de síntomas de bloqueo del pensamiento en aquellos pacientes con inicio precoz de la enfermedad. Estos pacientes se quejaban a menudo de tener "bloqueos de pensamiento " inesperados, como si su mente hubiera quedado en blanco, o como si los pensamientos le hubieran sido "robados". Es importante destacar que las correcciones efectuadas con respecto a la cronicidad sólo dieron lugar a mínimas modificaciones en cuanto a los hallazgos.

Aunque los descubrimientos son coherentes con las observaciones clínicas, los resultados no son significativos. El estudio se basa en un diseño retrospectivo, por lo que precisa 
de estudios prospectivos con el fin de examinar de una manera más completa las relaciones existentes entre la edad de inicio (E.I.) y los síntomas de la esquizofrenia, sin embargo, dada la gran eficacia de los últimos fármacos aparecidos, puede que los estudios prospectivos ofrezcan una visión bastante imprecisa de lo que podía esperarse de esta enfermedad.
LANDMARK, J., MERSKEY, H., CERNOVSKY, Z., \& HELMES, E. The positive triad of schizophrenic symptoms. British Journal of Psychiatry, 156, 388-394, 1990.

TORREY, F. The epidemiology of schizophrenia: questions needing answers. In: S.C. Schultz C.A. Tamminga (eds.), Schizophrenia: scientific progress. New York, Oxford University Press, pp. 45-51, 1989.

\section{Apéndice}

\section{Bibliografía}

ANGST, J., BAASTRUP, P.,GROF, P.,HIPPIUS, H.,POLDINGER, W., VARGA,E.,WEIS, P. \& WYES, F. Statistische Aspekte des Beginns und Verlaufs schizophrener Erkrankungen. In: G. Huber (editor), Verlauf und Ausgang schizophrener Erkrankungen. Stuttgart: F.K. Schattauer Verlag, 1973.

BLEULER, M. Die spactschizophrenen Krankheitsbilder. Fortschr. Neurol. Psychiatrie, 15, 259, 1943.

CERNOVSKY, Z.Z. \& LANDMARK, J. Correlates of hysterical symptoms in schizophrenic patients. Psycholo gical Reports, 75, 251-255, 1994.

CERNOVSKY, Z.Z., LANDMARK, J., HELMES, E. Are schizophrenic symptoms different in patients with higher education? Psychological Reports, 75,1552-1554,1994.

CERNOVSKY, Z.Z., LANDMARK, J., LESLIE, B. Social and anamnestic correlates of consensus in diagnosing schizophrenia. Journal of Clinical Psychology, 41, 614-619, 1985.

CERNOVSKY, LANDMARK, J. \& O'REILLY, R.L. Symptom patterns in schizophrenia for men and women. Psychological Reports, 80, 1267-1271, 1997.

HAMILTON, M. Fish's Schizophrenia Bristol (UK), London, Boston: Wright \& Sons Ltd, 1984.

HELMES, E., LANDMARK, J., \& KAZARIAN, S. Inter-rater reliability of 12 diagnostic systems of schizophrenia The Journal of Nervous and Mental Disease, 171, 307-311, 1983.

LANDMARK, J. A Manual for the Assessment of Schi zophrenia (Acta Psychiatrica Scandinavica, Supplementum 198, Vol. 65). Copenhagen: Munksgaerd, 1982.

LANDMARK, J.,CERNOVSKY, Z.Z.,MERSKEY, H., \& LESLIE, B. Interrelationships of systems for diagnosing schizophrenia Comprehensive Psychiatry, 27, 343-350. 1986.
Listado de los síntomas más importantes para el diagnóstico de esquizofrenia, utilizados en este trabajo. 88 son los síntomas que aparecen en este listado, aunque al igual que en otros estudios llevados a cabo por el equipo de Landmark (e.g, Cernovsky y Landmark 1994), el item 51 (aumento por amital sódico e.v.) no es válido, por lo que se redujo a un total de 87 variables.

1. Sonorización del pensamiento.

2. Voces que argumentan.

3 . Voces que comentan.

4. Percepción deliroide.

5. Pasividad somática.

6. Pensamientos "hechos".

7. Impulsos "hechos".

8. Actos volitivos "hechos".

9. Robo del pensamiento.

10. Inserción del pensamiento.

11. Pensamiento difuso.

12. Asociación.

13. Autismo.

14. Ambivalencia.

15. Afecto.

16. Síntomas catatónicos. 
17. Delusiones.

18. Alucinaciones.

19. Ruptura del desarrollo de la personalidad.

20. Sentimientos precoces.

21. Despersonalización y/o desrealización (Concepto de Langfeld).

22. Idea delirante primaria.

23. Apatía.

24. Conducta alterada o peculiar.

25. Negativismo.

26. Estereotipia persistente.

27. Alteración motora persistente.

28. Deterioro con el tiempo.

29. Volición.

30. Atención.

31. Manierismo bizarro.

32. Obediencia automática.

33. Ideas de referencia.

34. Alucinaciones auditivas.

35. Alucinaciones táctiles.

36. Otras alucinaciones.

37. Sentimientos de pasividad.

38. Aislamiento social.

39. Conciencia de enfermedad.

40. Juicio.

41. Delirios paranoides.

42. Neologismo.

43. Despersonalización y/o desrealización (Concepto usual).

44. Bloqueo del pensamiento.
45. Sintomatología polimorfa.

46. Alteraciones de la afectividad y fluctuaciones.

47. Aumento de estados parahipnóticos (Dongier).

48. Oscilación entre conciencia de enfermedad y manifestación delirante (Delirio).

49. Vuelta espontánea a un nivel premórbido.

50. Alta intensidad de la experiencia delirante.

51. Aumento por inyección endovenosa de Amytal Sódico.

52. Empobrecimiento de las relaciones.

53. Extensión de las ideas delirantes.

54. Lenguaje incoherente.

55. Información poco segura.

56. Ideas delirantes bizrras.

57. Ideas delirantes nihilistas.

58. Ideas delirantes hipocondríacas.

59. Ideas delirantes de grandiosidad.

60. Ideas delirantes místicas.

61. Ideas delirantes combinadas con alucinaciones.

62. Preocupación unida a delirios y/o alucinaciones.

63. Alucinaciones durante muchos días.

64. Trastornos del pensamiento combinados con embotamiento afectivo, ideas delirantes, alucinaciones o comportamiento desorganizado.

65. Síntomas obsesivo-compulsivos.

66. Otros síntomas esquizofreniformes.

67. Humor afectivo inestable y alegre. 
68. Alteración cuantitativa del discurso o lenguaje.

69. Cambios en la actividad motora (Aumento o disminución).

70. Humor depresivo (Que no responde a los cambios ambientales).

71. Dificultades en el pensamiento.

72. Curso fásico de la enfermedad.

73. Despertar precoz.

74. Cambios irreales en la autoestima personal.

75. Ideas delirantes de culpabilidad, de indignidad y de pecado.

76. Cambios fásicos en torno al apetito, peso o actividad sexual.

77. Facies depresiva.

78. Otros síntomas maniaco-depresivos.

79. Persistencia de delirios de persecución.
80. Ideas delirantes persistentes de celos.

81. Desorientación.

82. Trastornos de la memoria.

83. Confusión.

84. Síntomas neuróticos.

85. Síntomas histéricos.

86. Síntomas propios de los trastornos del personalidad.

87. Síntomas de trastornos sexuales.

88. Síntomas de otras enfermedades mentales.

Dirección para correspondencia:

Dr. Zack Cernovsky

Department of Psychiatry

Universit of Western Ontario

98 Greenbrier Crescent

London, Ontario

CANADA N6J 3X9 